\title{
Systematic assessment of difficult-to-treat asthma
}

\author{
D.S. Robinson*, D.A. Campbell ${ }^{\#}$, S.R. Durham ", J. Pfeffer", P.J. Barnes ${ }^{+}$, K.F. Chung ${ }^{+}$, for the Asthma \\ and Allergy Research Group of the National Heart and Lung Institute
}

Systematic assessment of difficult-to-treat asthma. D.S. Robinson, D.A. Campbell, S.R. Durham, J. Pfeffer, P.J. Barnes, K.F. Chung, for the Asthma and Allergy Research Group of the National Heart and Lung Institute. (C) ERS Journals Ltd 2003. ABSTRACT: Five per cent of asthmatics remain symptomatic despite high-dose treatment. The aim of the study was to investigate how often such difficult-to-treat asthma is due to intractable asthma, misdiagnosis, non-adherence with therapy, or psychiatric problems.

Difficult asthma was defined as persistence of symptoms despite treatment at step 4 of British guidelines or requirement for long-term oral glucocorticoids (step 5). Onehundred patients with a respiratory physician diagnosis of asthma were investigated in a single tertiary respiratory unit in an open and descriptive study.

Twelve of the patients studied did not have asthma and a further seven had additional diagnoses. Of the remainder, 55 had an asthma diagnosis confirmed by demonstration of reversible airflow narrowing or peak flow variability, whilst 20 did not. Noncompliance with prednisolone therapy was more frequent in the 55 with confirmed

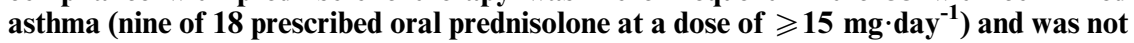
detected in the "unconfirmed asthma" group. There were no other significant differences between these groups. A major psychiatric component was detected in 10 patients.

Systematic evaluation of difficult asthma is useful as it can identify alternative or additional diagnoses, psychiatric illness or nonconcordance with therapy in a substantial proportion of cases $(32 \%$ in the present series).

Eur Respir J 2003; 22: 478-483.

\begin{abstract}
*Depts of Allergy, "Upper Respiratory Medicine, and ${ }^{+}$Thoracic Medicine, National Heart and Lung Institute, Faculty of Medicine, Imperial College and ${ }^{\#}$ Royal Brompton and Harefield NHS Trust, London, UK.
\end{abstract}

Correspondence: D.S. Robinson, Dept of Allergy and Clinical Immunology, National Heart and Lung Institute, Faculty of Medicine, Imperial College London, Dovehouse Street, London SW3 6LY UK.

Fax: 442073518135

E-mail: d.s.robinson@imperial.ac.uk

Keywords: Adherence, diagnosis, psychiatry, severe asthma

Received: February 142003

Accepted after revision: May 232003

D.A. Campbell is funded by the Joint Research Committee of the Royal Brompton and Harefield Trust and National Heart and Lung Institute. D.S. Robinson was funded in part by the Wellcome Trust.
Although widespread use of anti-inflammatory treatment has resulted in good control of asthma for many, an important subgroup of $5-10 \%$ of patients remain symptomatic despite treatment [1, 2]. These subjects include severe asthmatics who are at increased risk of dying from asthma and who have continued morbidity from both their disease and the oral corticosteroids that are often used to treat it [2, 3]. Previous descriptions of severe or "difficult" asthma have suggested clinical subgroups, including brittle asthma [4] and corticosteroid-insensitive asthma [5], and, in some cases, such classification may be a useful guide to management. Corticosteroid-sparing immunosuppressive agents, such as cyclosporin A and methotrexate, have been used for severe asthma, with variable success that may reflect the heterogeneity of disease [6, 7], as suggested by recent bronchoscopic studies and noninvasive measures of inflammation in severe asthma [8, 9]. In addition to disease heterogeneity it has been suggested that alternative diagnoses, nonadherence with therapy and psychiatric comorbidity, may contribute to difficulty in controlling asthma $[10,11]$.

This study focused on the practical problem of the patient with diagnosed asthma whose symptoms are not controlled despite high doses of inhaled corticosteroids and other regular therapy. The findings from a systematic assessment of 100 patients with difficult asthma are presented here. The data suggest that even in those patients referred to a tertiary centre for assessment there is a considerable rate of alternative or additional diagnoses, nonadherence with therapy and psychiatric comorbidity. Careful clinical assessment should identify these patients. A substantial minority did not have reversible airflow obstruction at the time of assessment.

\section{Methods}

\section{Patients}

Any patient with an asthma diagnosis from a respiratory physician referred to the Royal Brompton Hospital because of difficult asthma was eligible for inclusion in the study. Difficult asthma was defined as symptomatic asthma despite high-dose inhaled corticosteroid therapy $\left(>1,000 \mu \mathrm{g} \cdot \mathrm{day}^{-1}\right.$ of beclomethasone dipropionate or equivalent) and longacting $\beta_{2}$-agonist or theophylline treatment (step 4 of the British Thoracic Society Guidelines) [2], or patients treated with long-term ( $>3$ months) oral corticosteroids for asthma (step 5). Inclusion criteria were deliberately broad so as to include all referred patients and lack of reversibility to inhaled $\beta_{2}$-agonists or smoking were not exclusion criteria, because the authors wanted to describe those patients being treated for asthma (and to determine in how many there were other explanations for continued symptoms). Patients kept a 14-day diary of peak expiratory flow (PEF) and symptoms and were then were assessed over a 4-day stay at the hospital. The reason for admission to the minimal dependency ward was that many patients had travelled long distances to the centre; most of the investigations could also be performed in an outpatient setting. The data on the first 100 patients assessed using this protocol is presented here. 


\section{Questionnaires}

Patient history, triggers for asthma and treatment were recorded on a standardised history and examination sheet. A St George's Respiratory Questionnaire [12] and General Health Questionnaires (GHQ 30) [13] were also completed.

\section{Clinical assessment}

During the inpatient stay, patients were seen in the ear, nose and throat (ENT) clinic by a consultant psychiatrist and by two consultant respiratory physicians. The ENT specialist was asked about the presence of rhinosinusitis and vocal cord dysfunction (seen at the time of assessment). In addition to the GHQ 30 questionnaire, the psychiatrist used a grading system to assess psychiatric problems contributing to perception of asthma and those that were coincidental or not impacting on asthma severity. A major psychiatric component contributing to asthma severity was when patients reported that $\geqslant 30 \%$ of symptomatic episodes were related to feeling tense or said this was "why I get breathless". A minor contribution to asthma severity was noted when asthma started or got worse after a major life event, but the patient did not link asthma symptoms with stress. Similarly a minor contribution was scored if stress caused $<30 \%$ of episodes or if asthma symptoms that had been related to stress persisted after the stress resolved.

\section{Atopy}

Patients were specifically asked about allergic triggers for asthma symptoms and the presence of allergic rhinitis. In addition, skin-prick tests were performed for house dust mite, grass pollen, cat, dog and Aspergillus allergens using standardised extracts and positive and negative controls (ALK-Abello, Hungerford, UK), with a wheal $3 \mathrm{~mm}$ larger than the negative control being graded as positive. Total serum and allergenspecific immunoglobulin (Ig)E for cat, dog, grass pollen, house dust mite and Aspergillus were measured in the biochemistry laboratory using Phadebas radioallergosorbent test (RAST; Pharmacia, Milton Keynes, UK).

\section{Blood tests}

Full blood count, biochemistry and serum theophylline concentration (blood taken $6 \mathrm{~h}$ post-dose) were performed in the hospital routine laboratories and serum Aspergillus precipitins were also measured. Serum cortisol and prednisolone were measured by high- performance liquid chromatography (HPLC) in samples taken $2 \mathrm{~h}$ after dosing (Komtron HPLC 525 pump system; Komtron Instruments, Watford, UK).

\section{Imaging}

All patients had a limited protocol high-resolution computed tomogram with inspiratory and expiratory films (Imatron, San Francisco, CA, USA) in addition to a plain chest radiograph. Radiographs were reviewed by a consultant radiologist who graded the chest radiograph as normal, hyper-expanded, or abnormal, and the computed tomography (CT) scans as normal or showing air trapping, bronchial wall thickening, bronchiectasis, or other abnormality [14].

\section{Lung function tests}

All patients underwent full lung function tests, including spirometry, static lung volumes, conductance and gas transfer, performed in the lung function dept of the Royal Brompton Hospital. Bronchodilator therapy (including nebulised or subcutaneous treatment) was withheld for $\geqslant 8 \mathrm{~h}$ before testing and long-acting $\beta_{2}$-agonists were withheld for $\geqslant 24 \mathrm{~h}$. Reversibility to $\beta_{2}$-agonists was assessed in the lung function laboratory and separately by a physiotherapist on another day using a defined protocol involving increasing doses of salbutamol and ipratropium.

\section{Analysis}

All patients were reviewed after completion of the protocol and the diagnosis of asthma or non-asthma was made. Patients were further separated on the basis of those with confirmed reversible airflow obstruction and/or peak flow variability ( $>15 \%$ amplitude mean per cent on a 14-day home diary [15]) and those who had neither reversibility nor PEF variability at assessment (unconfirmed asthma).

All data were entered into a database by the research nurse and there were periodic data checks. Statistical analysis was performed. Data were analysed for normality by the RyanJoiner test, then expressed as mean \pm SD or median and range if not normally distributed. Comparison between confirmed asthma and unconfirmed asthma was made using an unpaired t-test or Mann-Whitney U-tests or by Fisher's exact-test analysis. A p-value of $<0.05$ was considered significant.

\section{Results}

One-hundred patients with difficult asthma were evaluated over 2 yrs. Of these, 92 were from hospital chest clinics and eight were new referrals from primary care. The inclusion of patients into different categories is summarised in figure 1.

\section{Alternative or additional diagnoses}

Twelve patients were judged to have diagnoses other than asthma because there was no evidence of, nor history suggestive of, reversible airflow obstruction, in conjunction with another explanation for their symptoms (table 1). Chronic obstructive pulmonary disease (COPD) was diagnosed on the basis of irreversible airflow obstruction at the time of assessment, with no previously documented reversibility, previous smoking and reduced gas transfer. One patient had emphysema on lung-function testing and CT scan and was $\alpha_{1}$-antitrypsin deficient. Another patient had cystic fibrosis (CF) with symptoms of breathlessness cough and sputum, bronchiectasis on chest radiograph and CT, Pseudomonas aeruginosa cultured from sputum and a positive $\mathrm{CF}$ genotype. Cardiomyopathy was suspected clinically in one 30-yr-old male and confirmed on echocardiography and cardiac biopsy. Obliterative bronchiolitis was diagnosed in a female with progressive breathlessness and CT features, respiratory muscle problems were diagnosed on diaphragm and mouth pressure measurements, and severe anxiety diagnosed by the psychiatrist was accompanied by paradoxical vocal cord movement at ENT examination.

A further group of respiratory diagnoses coexistent with asthma were made (fig. 1). The most common was bronchiectasis. 


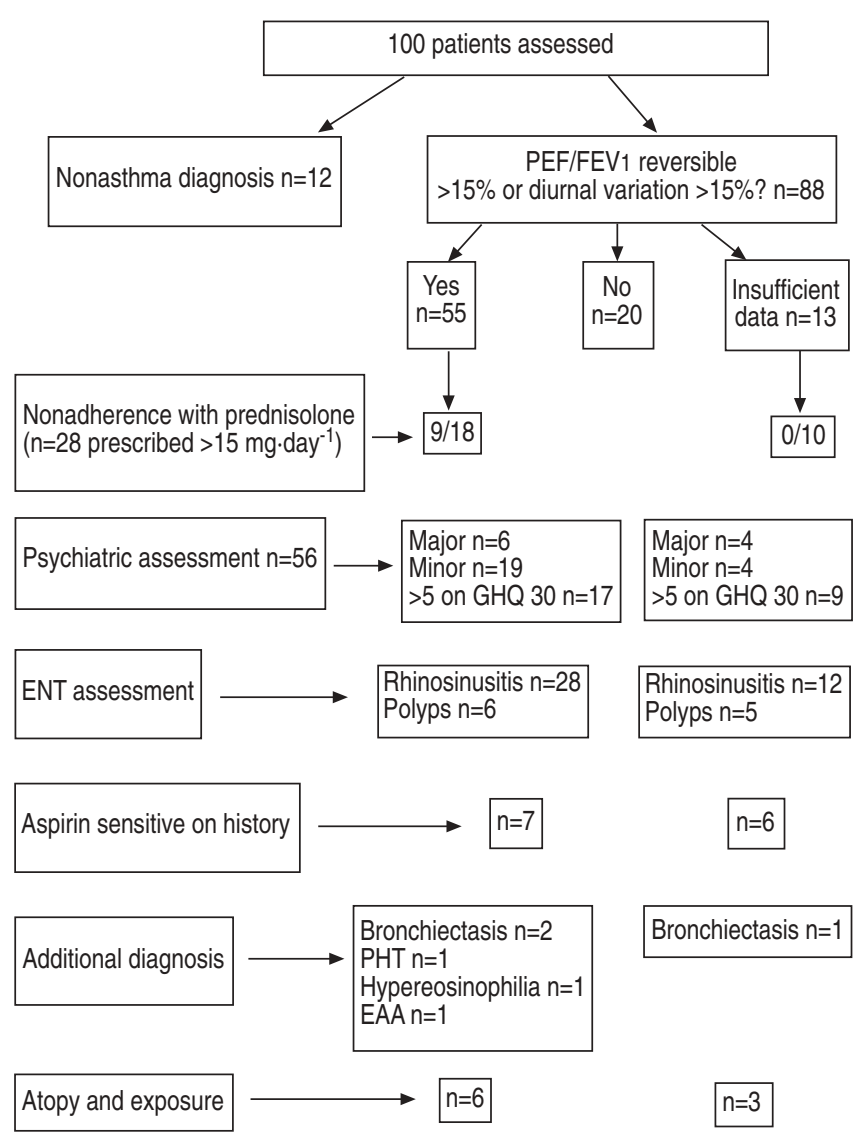

Fig. 1.- Flow chart showing numbers of patients in each category. All 75 patients thought to have asthma were assessed for ear, nose and throat (ENT) disease, atopy history of aspirin sensitivity and additional diagnoses. Prednisolone levels were measured in patients taking oral prednisolone and were judged as a useful measure of nonadherence if serum prednisolone was undetectable or serum cortisol was normal in patients prescribed $\geqslant 15 \mathrm{mg} \cdot \mathrm{day}^{-1}$. PEF: peak expiratory flow; FEV1: forced expiratory volume in one second; GHQ 30: General Health Questionnaire; PHT: pulmonary hypertension; EAA: extrinsic allergic alveolitis.

\section{Table 1.-Diagnoses in patients without asthma}

Chronic obstructive pulmonary disease

Emphysema ( $\alpha_{1}$-antitrypsin deficient)

Cystic fibrosis

Cardiomyopathy

Obliterative bronchiolitis

Respiratory muscle incoordination

Severe anxiety and vocal cord dysfunction

\section{Lung function tests and symptoms}

Lung function data are shown in table 2. After excluding those patients in whom a diagnosis other than asthma was made, reversibility testing and peak flow data was available in 75. Of these, 55 had a reversible airflow obstruction of $\geqslant 15 \%$ or peak flow variability, confirming asthma at the time of the assessment, whilst 20 had neither peak flow variability nor reversibility to bronchodilators. These 20 without confirmed reversibility are termed "unconfirmed asthma". A further 13 patients were excluded from analysis because, although reversibility was not seen during assessment, they did not return the peak flow charts so the authors were unable to classify them as confirmed or unconfirmed asthma. All
Table 2.-Patient details, lung function, quality of life and atopic status in asthmatics

\begin{tabular}{|c|c|c|}
\hline & $\begin{array}{l}\text { Confirmed } \\
\text { asthma }\end{array}$ & $\begin{array}{l}\text { Unconfirmed } \\
\text { asthma }\end{array}$ \\
\hline Subjects n & 55 & 20 \\
\hline Age yrs & $36 \pm 12.5$ & $47.7 \pm 15.2$ \\
\hline Sex M:F & $14: 41$ & 9:11 \\
\hline \multicolumn{3}{|l|}{ Age at onset yrs } \\
\hline$<20$ & 38 & 12 \\
\hline$>20$ & 17 & 8 \\
\hline Ventilated & 11 & 3 \\
\hline Smokers & 8 & 2 \\
\hline FEV1 \% & $63.6 \pm 23.9$ & $66.4 \pm 22.8$ \\
\hline $\mathrm{FVC} \%$ & $86.6 \pm 17$ & $89.6 \pm 20.2$ \\
\hline TLC \% & $107.2 \pm 16.4$ & $105.3 \pm 17.9$ \\
\hline$T \mathrm{~L}, \mathrm{CO} \%$ & $90.5 \pm 19.3$ & $91.9 \pm 21.6$ \\
\hline sGaw \% & $61.8 \pm 57.5$ & $58.9 \pm 26.6$ \\
\hline PEF \% & $63.3 \pm 17.3$ & $70.9 \pm 22.4$ \\
\hline Amplitude mean \% & $27.3 \pm 18.4$ & $10.9 \pm 4.0$ \\
\hline \multicolumn{3}{|l|}{ Blood gases } \\
\hline $\mathrm{H}+$ & $34.5 \pm 5.51$ & $34 \pm 3.8$ \\
\hline $\mathrm{Pa}, \mathrm{CO}_{2}$ & $4.52 \pm 0.61$ & $4.43 \pm 0.9$ \\
\hline $\mathrm{Pa}, \mathrm{O}_{2}$ & $10.89 \pm 2.58$ & $10.82 \pm 2.9$ \\
\hline $\mathrm{HCO}_{3}$ & $23.04 \pm 2.45$ & $23.2 \pm 2.9$ \\
\hline \multicolumn{3}{|l|}{ SGRQ scores } \\
\hline Total & $61.8 \pm 17.3$ & $58.1 \pm 21.9$ \\
\hline Symptoms & $82.7 \pm 12.5$ & $75.8 \pm 12.8$ \\
\hline Activities & $68.8 \pm 25.3$ & $68.8 \pm 28.6$ \\
\hline Impact & $51.6 \pm 20.1$ & $48.2 \pm 22.0$ \\
\hline GHQ 30 questionnaire & $2.5(0-27,17 \geqslant 5)$ & $5.5(0-25,9 \geqslant 5)$ \\
\hline \multicolumn{3}{|l|}{ Atopy } \\
\hline Atopic & 39 & 11 \\
\hline \multicolumn{3}{|l|}{ Total IgE } \\
\hline Atopics & $145(20-8300)$ & $314(10-2620)$ \\
\hline Nonatopics & $47(2-252)$ & $24(7-112)$ \\
\hline House dust mite sensitised & $30 \pm 38$ & $8 \pm 10$ \\
\hline Grass pollen sensitised & $21 \pm 33$ & $10 \pm 10$ \\
\hline Cat sensitised & $18 \pm 19$ & $4 \pm 2$ \\
\hline Dog sensitised & $13 \pm 14$ & $2 \pm 2$ \\
\hline Aspergillus IgE & 7 & 4 \\
\hline
\end{tabular}

Data are presented as mean $\pm \mathrm{SD}$, except age at onset, whether ventilated and smoking status (numbers in each category). Lung function is shown as per cent predicted values (using European Respiratory Society predicted tables for height and age) and amplitude mean per cent peak expiratory flow (PEF) over a 14-day period was calculated as described in [16]. St George's Respiratory Questionnaire (SGRQ) scores are percentages (with high scores indicating impaired quality of life) and General Health Questionnaire (GHQ 30) is an arbitrary score for which a score of $\geqslant 5$ is indicative of "psychiatric caseness". M: male; F: female; FEV1: forced expiratory volume in one second; FVC: forced vital capacity; TLC: total lung capacity; TL,CO: transfer coefficient of the lung for carbon monoxide; sGaw: specific airway conductance; $\mathrm{Pa}, \mathrm{CO}_{2}$ : arterial carbon dioxide tension; $\mathrm{Pa}, \mathrm{O}_{2}$ : arterial oxygen tension; IgE: immunoglobulin E.

patients reported a considerable burden of symptoms with cough, wheeze, and breathlessness. Twenty-three patients reported daily sputum production.

\section{Atopy, aspirin sensitivity and ear, nose and throat assessment}

The frequency of atopic sensitisation is shown in table 2 . Seven patients sensitised to cats continued to keep cats and four of those sensitised to dogs had a dog at home. ENT disease and history of aspirin sensitivity is summarised in figure 1. 


\section{Psychiatric evaluation and quality of life impact}

Quality of life scores for those with asthma are shown in table 2. Psychiatric assessment is shown in figure 1.

\section{Treatment and drug levels: adherence with treatment}

Treatment at the time of assessment is shown in table 3. All patients were prescribed high doses of inhaled corticosteroids. In addition to the 52 patients on continuous oral prednisolone, a further 27 had received at least two short courses of oral corticosteroids in the past year.

Serum prednisolone and cortisol concentrations were available from 18 of 23 patients with airflow reversibility and 10 of 12 without reversibility at assessment who were prescribed $\geqslant 15 \mathrm{mg}$ daily oral prednisolone (fig. 1 and table 3). Serum prednisolone was undetectable in six of those with reversibility at the time of assessment (with normal serum cortisol in these patients and three others suggesting nonadherence with therapy), but all of those without confirmed asthma taking $\geqslant 15 \mathrm{mg} \cdot \mathrm{day}^{-1}$ of prednisolone had detectable serum drug and suppressed cortisol $(\mathrm{p}<0.05$ by Fisher's exact test).

\section{Radiology}

Chest radiographs were normal in 45 of those with confirmed asthma and 16 patients with unconfirmed asthma. Four radiographs showed hyper-expanded lung fields (all unconfirmed asthma), four changes of bronchiectasis, five linear shadowing, one emphysema and one pulmonary arterial enlargement. CT was normal in 13 of the patients with reversibility or PEF variability and in 15 of those with unconfirmed asthma. Seven patients had frank bronchiectatic changes on CT (six also had confirmed asthma), one had consolidation suggestive of extrinsic allergic alveolitis, one changes consistent with obliterative bronchiolitis and one features of emphysema.

\section{Discussion}

The descriptive data for 100 patients assessed at the Royal Brompton Hospital for difficult-to-treat asthma is presented here. There were high rates of alternative or additional diagnoses to asthma, nonadherence with therapy, psychiatric comorbidity and considerable impairment of quality of life. In addition, many patients did not have any peak flow variability or reversible airflow obstruction at the time of assessment.

The authors deliberately set broad inclusion criteria as it was their intention to describe patients currently being treated for severe asthma. Twelve patients had non-asthma diagnoses after systematic review and a number of these diagnoses had important management implications which led to subsequent changes in treatment. These findings emphasise the importance of systematic investigation and review of the asthma diagnosis in those with difficult or apparently corticosteroidinsensitive asthma [10, 11, 17]. The authors suggest the following approach to asthmatic patients not responding to treatment. First, a careful review of symptoms may reveal chronic sputum production or smoking suggesting either of COPD or bronchiectasis, which may then be confirmed by radiology, bacteriology or lung function tests. In those patients taking oral theophylline or prednisolone measurement of blood levels or serum cortisol is a useful indicator of possible nonadherence. A social and psychiatric history may reveal underlying problems affecting the interpretation of symptoms. Documentation of reversibility and diurnal variation of airflow obstruction may identify fixed airflow obstruction, although how this should be managed requires further research. Often a "fresh" opinion from a colleague or tertiary centre will help with these patients, but the authors suggest that a structured approach including all of these components should be taken.

Many studies with strict inclusion criteria of currently demonstrable reversibility would have excluded nearly onethird of the remaining patients in whom no alternative explanation for symptoms was found. Nine of these 20 unconfirmed asthmatics had previously documented reversible airflow obstruction, either to $\beta_{2}$-agonists or oral corticosteroids.

Despite long-acting $\beta_{2}$-agonists, theophylline and nebulised

Table 3. - Treatment at time of assessment and adherence with prednisolone and theophylline treatment

Confirmed asthma

Unconfirmed asthma

\begin{tabular}{|c|c|c|}
\hline Subjects $n$ & 55 & 20 \\
\hline BDP & $1(2000)$ & 0 \\
\hline BUD & $23(2000,800-12000)$ & $6(1800,1000-3200)$ \\
\hline FP & $32(2000,1000-4000)$ & $14(1500,1000-3000)$ \\
\hline LAB & 37 & 15 \\
\hline Theophylline & 25 & 9 \\
\hline Undetectable in serum & 2 & 2 \\
\hline \multicolumn{3}{|l|}{ Nebulised } \\
\hline$\beta_{2}$-agonist & $28(+18$ as required $)$ & $8(+2$ as required $)$ \\
\hline \multicolumn{3}{|l|}{ Subcutaneous } \\
\hline Terbutaline & $11(5,2-12)$ & $1(12)$ \\
\hline Prednisolone & $38(15,12.5-125)$ & $14(30,2-60)$ \\
\hline Serum level undetectable* with normal cortisol & $6 / 18$ & $0 / 10$ \\
\hline Serum level detectable with normal cortisol & $3 / 18$ & $0 / 10$ \\
\hline \multicolumn{3}{|l|}{ Mean concentration ${ }^{\#} \mu \mathrm{g} \cdot \mathrm{L}^{-1}$} \\
\hline$>15 \mathrm{mg} \cdot \mathrm{day}^{-1}$ & $616 \pm 143$ & $624 \pm 125$ \\
\hline$<15 \mathrm{mg} \cdot \mathrm{day}^{-1}$ & $465 \pm 90$ & $397 \pm 136$ \\
\hline
\end{tabular}

Patients were divided into those with confirmed asthma (history and/or either bronchodilator reversibility or diurnal variation on peak expiratory flow (PEF) charts) and unconfirmed asthma (history but not currently reversible or with peak flow variability). Beclomethasone dipropionate (BDP), budesonide (BUD) and fluticasone propionate (FP) are all shown as median dose in $\mu \mathrm{g} \cdot \mathrm{day}^{-1}$ (range). Long-acting $\beta_{2}$-agonist (LAB), theophylline, nebulised $\beta_{2}$-agonist, subcutaneous terbutaline and oral prednisolone are shown as numbers in each group taking each treatment (with median dose and range in $\mathrm{mg} \cdot \mathrm{day}^{-1}$ for subcutaneous terbutaline and prednisolone). Adherence with prednisolone is shown for patients prescribed $\geqslant 15 \mathrm{mg} \cdot$ day $^{-1}$ (should have suppressed cortisol). ${ }^{\#}$ : data presented as mean+SE; *: $\mathrm{p}<0.05$. 
therapy being omitted on the day of assessment of $\beta_{2}$-agonist response, it is possible that the persistent bronchodilator effect of such therapy masked response. Similarly, a systematic trial of high-dose oral corticosteroids in patients without PEF variability of bronchodilator responsiveness was not performed because many were already treated with corticosteroids.

No relationship between the presence or absence of reversible airflow obstruction at the time of assessment to any features of asthma severity, radiological changes, other lung function measures or quality of life measures was found. It worth noting that previous study of persistent airflow limitation in severe asthma found an association with adult onset, airway hyperresponsiveness, and sputum eosinophilia [16].

Noncompliance with oral corticosteroid therapy was confirmed in nine out of 18 patients with asthma prescribed $>15 \mathrm{mg} \cdot \mathrm{day}^{-1}$ prednisolone. All 10 of those without confirmed asthma at the time of assessment who were prescribed $>15 \mathrm{mg} \cdot \mathrm{day}^{-1}$ of prednisolone were compliant. The reason for this difference in adherence is not known. It is possible that the steroids had reversed any reversible component of airflow obstruction in these patients, but also that these patients had themselves noticed clinical improvement with steroid therapy. Further studies of rates of steroid response in severe asthma are required. Corticosteroid resistance in these patients was not formally assessed, although previous studies suggest that this may be a reason for failure to respond to corticosteroids in a small proportion of severe asthmatics [7, 5, 18]. A similar protocol has been used in the authors' institute for the assessment of difficult asthma in children, and this did suggest that a subgroup of patients had steroid insensitivity [19]. Nonetheless, the data suggest that nonadherence with therapy may be a more common explanation.

Four of the patients with unconfirmed asthma had normal lung function (and two had no evidence of bronchial hyperresponsiveness). Nonetheless, two of these patients had been ventilated in the past for asthma and were thought to have type- 2 brittle asthma (precipitous falls in PEF on the background of normal lung function). Another two were judged to have a major psychiatric component to their asthma symptoms. It is difficult to confidently diagnose type-2 brittle asthma without documented episodes of severe airflow obstruction, but the diagnosis cannot be excluded if there is a history of previous hospital admissions. It is also uncertain what regular therapy is useful for type- 2 brittle asthma, since lung function is relatively normal between attacks [4]. Larger patient databases will be required to study this important group who appear to be at risk of dying from asthma.

The patients studied had been referred to a tertiary referral centre. It is possible that the present findings may underestimate non-asthma diagnoses, nonadherence or significant psychiatric disturbance since these patients may not be referred to a tertiary centre. In addition, the findings may underestimate conditions mimicking asthma that cause an acute presentation, such as vasculitis.

In the present patient population, 10 were thought to have a major psychiatric component to their symptoms. Even if this is an under-representation of psychiatric disease in difficult asthma, because of selective referral, the data argue that it is important to investigate difficult asthmatics thoroughly and not to dismiss their problems [20]. Ear lobe blood gas analysis was performed in all patients as part of lung function assessment, and in 11 patients whose symptoms were suggestive of hyperventilation it showed a resting arterial carbon dioxide tension of $<4 \mathrm{kPa}$. It may be that hyperventilation questionnaires will be helpful in assessing difficult-to-treat asthma and the authors are currently evaluating such a tool. Those with identified psychiatric morbidity need careful multidisciplinary management to try to address their underlying problems.

Over one-half of the asthmatics studied had rhinosinusitis at ENT assessment. Treatment of upper airway disease is important and, although controlled trials are required, may improve asthma control [21, 22]. Fifty were atopic (as judged by at least one positive skin-prick test to aeroallergens). Total serum IgE was normal in a number of those with atopy, emphasising that this is not a useful screen. Previous data from TUNNICLIFFE et al. [23] suggest that atopy is not more common amongst severe asthmatics compared to those with mild disease, but exposure to the allergen to which they are sensitised is greater in those with severe disease [23]. It is worth noting that patients' perception of allergen sensitisation was not always related to detection of specific IgE, emphasising the importance of skin-prick tests or RAST to confirm allergen sensitisation, although this should always be assessed in the context of the clinical history. Thirteen of the subjects reported aspirin sensitivity. There is little data available on the rate of aspirin sensitivity in UK asthmatics, but the European Network For the Understanding of Mechanisms Of Severe Asthma (ENFUMOSA) study identified an increased rate of aspirin sensitivity in severe asthmatics [10].

In conclusion, this systematic protocol for assessment of difficult asthma identified a significant proportion of patients $(32 \%)$ with other or additional respiratory disease, and other patients with psychiatric morbidity or nonadherence with therapy. Patients without demonstrable airflow obstruction may have residual irreversible obstruction not amenable to therapy or may have asthma triggered by allergens or salicylate that is not apparent on inpatient assessment. Those with measurable reversibility despite treatment have high noncompliance rates. Further work is required to correlate clinical and inflammatory phenotypes of asthma with treatment response. A national or international database of severe asthma may allow such studies to be undertaken and studies currently underway in the European Union and USA may provide such data.

\begin{abstract}
Acknowledgements. The authors would like to thank S.R. Durham and I.S. Mackay for ear, nose and throat assessment, J.M. Pfeffer for psychiatric assessment and D. Carr for radiological scoring. They are grateful to the Lung Function unit (D. Cramer), physiotherapists and staff on Lind Ward at the Royal Brompton for their help with care of these patients. They would also like to thank J. Matthews and W.J. Oldfield for comments on the manuscript.

Other members of the Asthma and Allergy Research Group of the National Heart and Lung Institute (London, UK): I. Adcock M. Belvisi, P. Cullinan, M. Giembycz, T. Hansel, P. Jeffery, S. Johnston, M. Jones, A.B. Kay, M. Larche, A. Newman Taylor, D. Rogers and P. Openshaw.
\end{abstract}

\section{References}

1. British Thoracic Society, British Paediatric Society, Research Unit of Royal College of Physicians of London, King's Fund Centre, National Asthma Campaign. The British Guidelines on Asthma Management: 1995 Review and Position Statement. Thorax 1997; 52: S1-S21.

2. Barnes PJ, Woolcock AJ. Difficult asthma. Eur Respir $J$ 1998; 12: 1209-1218.

3. Wareham NJ, Harrison BD, Jenkins PF, Nicholls J, Stableforth DE. A district confidential enquiry into deaths due to asthma. Thorax 1993; 48: 1117-1120. 
4. Ayres JG, Miles JF, Barnes PJ. Brittle asthma. Thorax 1998; 53: 315-321.

5. Szefler SJ, Leung DY. Glucocorticoid-resistant asthma: pathogenesis and clinical implications for management. Eur Respir J 1997; 10: 1640-1647.

6. Shiner RJ, Nunn AJ, Chung KF, Geddes DM. Randomised, double-blind, placebo-controlled trial of methotrexate in steroid-dependent asthma. Lancet 1990; 336: 137-140.

7. Alexander AG, Barnes NC, Kay AB. Trial of cyclosporin in corticosteroid-dependent chronic severe asthma. Lancet 1992; 339: 324-328.

8. Wenzel SE, Schwartz LB, Langmack EL, et al. Evidence that severe asthma can be divided pathologically into two inflammatory subtypes with distinct physiologic and clinical characteristics. Am J Respir Crit Care Med 1999; 160: 1001-1008.

9. Jatakanon A, Uasuf C, Maziak W, Lim S, Chung KF, Barnes PJ. Neutrophilic inflammation in severe persistent asthma. Am J Respir Crit Care Med 1999; 160: 1532-1539.

10. Proceedings of the ATS Workshop on Refractory Asthma. Current understanding, recommendations, and unanswered questions. Am J Respir Crit Care Med 2001; 162: 2341-2351.

11. Chung KF, Godard P, Adelroth E, et al. Difficult/therapy resistant asthma: the need for an integrated approach to define clinical phenotypes, evaluate risk factors, understand pathophysiology and find novel therapies. ERS Task Force on Difficult/Therapy-resistant asthma. Eur Respir J 1999; 13: 1198-1208.

12. Jones PW, Quirk FH, Chloe M, Baveystock M, Littlejonhs PA. Self-complete measure of health status for chronic airflow limitation. The St. George's Respiratory Questionnaire. Am Rev Respir Dis 1992; 145: 1321-1327.

13. Goldberg D, Rickels K, Dowing R, Hesbacher P. A comparison of two psychiatric screening tests. $\mathrm{Br} J$ Psych 1976; 129: 61-67.
14. Carr DH, Hibon S, Rubens M, Chung KF. Peripheral airways obstruction on high-resolution computed tomography in chronic severe asthma. Respir Med 1998; 92: 448453.

15. Higgins BG, Britton JR, Chinn S, et al. The distribution of peak expiratory flow variability in a population sample. Am Rev Respir Dis 1989; 146: 1368-1372.

16. ten Brinke A, Zwinderman AH, Sterk PJ, Rabe KF, Bel EH. Factors associated with persistent airflow limitation in severe asthma. Am J Respir Crit Care Med 2001; 164: 744-748.

17. Chan MTS, Leung DYM, Szefler SJ, Spahn JD. Difficult to control asthma: clinical characteristics of steroid insensitive asthma. J Allergy Clin Immunol 1998; 101: 594-601.

18. Corrigan CJ, Brown PH, Barnes NC, et al. Glucocorticoid resistance in chronic asthma. Glucocorticoid pharmacokinetics, glucocorticoid receptor characteristics, and inhibition of peripheral blood $\mathrm{T}$ cell proliferation by glucocorticoids in vitro. Am Rev Respir Dis 1991; 144: 1016-1025.

19. Payne DN, Wilson NM, James A, Hablas H, Agrafioti C, Bush A. Evidence for different subgroups of difficult asthma. Thorax 2001; 56: 345-350.

20. Miles JF, Garden GM, Tunnicliffe WS, Cayton RM, Ayres JG. Psychological morbidity and coping skills in patients with brittle and non-brittle asthma: a case-control study. Clin Exp Allergy 1997; 27: 1151-1159.

21. Fireman P. Rhinitis and asthma connection: management of coexisting upper airway allergic diseases and asthma. Allergy Asthma Proc 2000; 21: 45-54.

22. Bousquet J, Van Cauwenberge P. Allergic rhinitis and its impact on asthma (ARIA). J Allergy Clin Immunol 2001; 108: S1-S147.

23. Tunnicliffe WS, Fletcher TJ, Hammond K, et al. Sensitivity and exposure to indoor allergens in adults with differing asthma severity. Eur Respir J 1999; 13: 654-659. 